\title{
SURGICAL TECHNIQUE GAN PREVENT ARM LYMPHOEDEMA AFTER LEVEL III CLEARANCE FOR BREAST CANCER
}

\author{
Ghosh SK', Burman DR
}

${ }^{1}$ Department of Surgical Oncology, ${ }^{2}$ Department of Laboratory Oncology (Oncopathology), Medical College, Kolkata

\begin{abstract}
Clearance of the axillary tissue during operation is still the mainstay of treatment for node positive breast cancer. Level III axillary nodal clearance is supposed to increase the risk of lymphoedema of arm, along with other factors. However, preservation of the fascia over the axillary vein during surgery reduces the risk of lymphoedema greatly. In this study we measured the incidence of arm lymphoedema that occured after Level III axillary clearance for breast cancer. During surgery, dissection over the anterior surface of axillary vein was limited to preserve the fascia covering axillary vein. Other factors commonly implicated in the development of post-operative arm lymphoedema were also documented and their effect analysed. Forty three patients underwent operation for breast cancer including complete axillary clearance up to Level III. The incidence of lymphoedema was $25.5 \%$ (11 out of 43 patients). None of these patients had severe lymphoedema. On multivariate analysis, no other associated factors like BMI, chemotherapy and nodal metastases had any bearing on the development of lymphoedema. We conclude that Level III axillary clearance of axilla is safe and not excessively morbid in terms of developing arm lymphoedema provided the fascia over axillary vein is preserved.
\end{abstract}

\section{KEYWORDS}

Complete axillary clearance, breast cancer, lymphoedema, fascia over axillary vein

\section{CORRESPONDING AUTHOR}

Dr. Saurav Kumar Ghosh,

Associate professor

Department of Oncosurgery, Medical College Kolkata

88 College Street, Kolkata, India- 700073

Email: sg00@rediffmail.com

ORCID ID: 0000-0001-5599-0484 


\section{INTRODUCTION}

The incidence of symptomatic lymphoedema of the arm following axillary clearance ranges widely from 15 to $33 \% .{ }^{1}$ The aetiology of arm lymphoedema is not clearly understood and is multifactorial but one important risk factor is the extent of axillary surgery. ${ }^{2}$ To reduce the morbidity of axillary dissection, several innovations in surgery like Sentinel node biopsy are now standard in most European countries and in the US. European guidelines for surgical treatment of breast cancer usually only recommend that axillary clearance be done up to Level II to reduce the chance of developing lymphoedema. However, in our country, the breast cancers are more advanced and more likely to be node positive. The incidence of positivity of Level III nodes is also much higher than in the West, hence cancer institutes in India usually do a complete axillary clearance including Level III. Major lymphatic channels of the arm pass through the axilla around the axillary vessels. Stripping of the fibrous sheath of the axillary vein during surgery is the important cause of developing lymphoedema, rather than the extent of nodes removed. To test this hypothesis, we conducted this observational study in our department.

The aim of this study is to assess the incidence of lymphoedema in the affected arm of breast cancer patients, following axillary clearance preserving the fascia over the axillary vein, as part of Modified radical mastectomy or Breast conservation surgery. We also aim to correlate the degree of lymphoedema with known risk factors for lymphoedema development like obesity, chest wall and axillary radiation, tumour size, number of positive nodes and pre-operative chemotherapy.

\section{MATERIALS AND METHODS}

This was a prospective observational study conducted in our institute by the Department of Surgical Oncology. All breast cancer patients who underwent definitive surgery for breast cancer in the Department of Surgical Oncology of our hospital from May 2013 onwards were included in the study. Informed written consent was taken from each patient about their willingness to participate in the study. Institutional ethics committee clearance was obtained for the study. The measurement of both upper limbs were recorded on the day before surgery and 1 year after surgery at 2 points as per a standard method. ${ }^{3}$ The measurements were taken at 11.5 cms above the olecranon in the arms and $7.5 \mathrm{cms}$ below the olecranon in the forearm keeping both arms flexed at $90 \%$ at the elbow. A prospective data base was maintained. Additional relevant information like body mass index, tumour size, neo-adjuvant chemotherapy, number of nodes positive on histopathology and administration of breast or axillary radiation were also recorded. Lymphoedema was considered mild if there was increase in limb circumference by $1 \mathrm{~cm}$ from the baseline value and significant if the change in circumference was more than $3 \mathrm{~cm}$ at any site. No compression device or garments were prescribed to any of these patients prophylactically unless significant lymphoedema was documented. All patients were counselled about care of affected arm and shoulder exercises. Exclusion criteria were previous surgery to either axillae or arms, patients with chronic renal failure, congestive cardiac failure or hypoproteinemia, those with proven recurrence in the axilla within the period of study, patients of proven connective tissue disorders and patients unwilling to follow up regularly. Data were tabulated in Excel sheet and analysis was done using Excel MS Office 8. Univariate analysis was done using Chi square Test and Multivariate analysis of the risk factors was done using Cox Logistic regression analysis.

\section{RESULTS}

Forty eight patients of breast cancer were included in this study from February 2013 to September 2015. However, 5 patients were lost to follow up so complete data from 43 patients were analysed. All were females. The age ranged from 32 years to 71 years with a median age of 56 years. The relevant patient and tumour characteristics are listed in Table 1.

Table 1: Patient and tumour characteristics

Total number of patients 43

Age range (Years) $32-71$

T1 tumours 0

T2 tumours 20

T3 tumours 10

T4 tumours 13

Axillary Nodal metastases present $\quad 19$ Axillary Nodal metastases absent 24 $\mathrm{BMI}>25 \mathrm{~kg} / \mathrm{m}^{2} \quad 26$ $\mathrm{BMI}<25 \mathrm{~kg} / \mathrm{m}^{2}$ 17

Twenty out of the 43 patients that is $46.5 \%$ patients had a T2 tumour (less than $5 \mathrm{cms}$ without any involvement of skin or chest wall). The remaining $53.5 \%$ patients all had $\mathrm{T} 3$ or $\mathrm{T} 4$ disease by virtue of presenting diameter being more than $5 \mathrm{~cm}$ or because of skin involvement. None of the patients in our series had a tumour less than $2 \mathrm{~cm}$ size. We calculated the Body Mass Index (BMI) of 
all patients. Using the WHO recommendation of normal BMI range being 18.5 to $25 \mathrm{~kg} / \mathrm{m}^{2}, 26$ out of the 43 patients $(60.46 \%)$ were overweight, having BMI more than $25 \mathrm{~kg} / \mathrm{m}^{2}$. The details of treatment given to the patients is shown in Table 2 .

\begin{tabular}{|lll|}
\hline \multicolumn{3}{|c|}{ Table 2: Treatment details } \\
1. & Treatment given & $\mathbf{n}$ \\
& Surgery & \\
& Modified Radical Mastectomy & 36 \\
& Breast Conservation Surgery & 07 \\
2. & Adidical Mastectomy & 00 \\
& No Adjuvant Radiotherapy & 27 \\
3. & Neo-adjuvant chemotherapy & 16 \\
& Adjuvant chemotherapy & 43 \\
\hline
\end{tabular}

All but 7 out of the 43 patients underwent modified radical mastectomy. The remaining 7 had breast conservation surgery. None of the patients required Radical mastectomy. Three patients had reconstruction, 2 using free TRAM flap and one using a pedicled LD myo-cutaneous flap. A complete axillary clearance including level III axillary nodes was done in all patients. Level III nodes were removed separately via the interpectoral route. The axillary vein was identified and dissection over its anterior surface was carefully avoided, so as to preserve the fascia over the vein. The number of nodes obtained from axilla ranged from 10 to 19 nodes with a mean of 14.5 nodes. Out of 43 patients, 19 were node positive (44.1\%). The number of involved nodes varied from 1 to 10 (mean 3.5, median 4). Adjuvant radiation was administered to all patients with $\mathrm{T} 3$ or $\mathrm{T} 4$ tumours, those with 3 or more axillary nodal metastases or those with peri nodal extension and all patients who had undergone Breast conservation surgery. Radiation was given to the chest wall and ipsilateral supra-clavicular fossa. Axillary radiation was not needed in any of the patients. Twenty seven patients out of the 43 (62.7\%) received adjuvant radiotherapy up to 45 Gray with a further tumour bed boost of 15 days. All patients received adjuvant chemotherapy as they were all at least T2 onwards. Fourteen patients (32.5\%) received neo-adjuvant chemotherapy. Hormone therapy was given according to standard NCCN guidelines for those patients who were Estrogen receptor/Progesterone receptor positive.

Twenty eight out of 43 patients showed some increase in the limb circumference at the level of either the mid-arm or mid-forearm at one year. However, according to our set criteria, only 11 out these 43 patients had an increase of more than $1 \mathrm{~cm}$. So the incidence of lymphoedema in our series was 11 out of $43(25.5 \%)$. None of the 43 patients in our series had an increase in arm or forearm circumference more than $3 \mathrm{~cm}$. Hence the incidence of significant lymphoedema of the arm at 1 year in our series was $0 \%$. We assessed the relation between lymphoedema and patient related variables like BMI, Tumour size and number of involved nodes and treatment related factors like radiotherapy to the breast and neo-adjuvant chemotherapy. Both univariate and multivariate analysis were done. On univariate analysis using Chi square test, tumour size greater than $5 \mathrm{~cm}$, axillary nodal involvement and adjuvant radiation to the breast were found to correlate with development of oedema. However on multivariate analysis, none of the factors were found to be predictive of development of lymphoedema. Table 3 shows the univariate analysis of the variables assessed for development of arm oedema.

Table 3: Results of analysis of risk factors for arm Lymphoedema (Univariate)

\begin{tabular}{|llcc|} 
S.No & Variable & Lymphoedema (\%) & p value \\
1. & Body mass index $>25 \mathrm{~kg} / \mathrm{m}^{2}$ & $6 / 26$ patients $(23.0 \%)$ & 0.34 \\
& Body mass index $<25 \mathrm{~kg} / \mathrm{m}^{2}$ & $5 / 17$ patients $(29.4 \%)$ & \\
2. & T1/T2 tumours & $4 / 20$ patients $(20.0 \%)$ & 0.04 \\
& T3/T4 tumours & $7 / 23$ patients $(30.4 \%)$ & 0.03 \\
3. & Node positive & $6 / 19$ patients $(31.5 \%)$ & 0.55 \\
4. & Rode negative & $5 / 24$ patients $(20.8 \%)$ & \\
5. & No neo-adjuvant chemo & $4 / 14$ patients $(28.5 \%)$ & 0.008 \\
\hline
\end{tabular}




\section{DISCUSSION}

Some swelling of the ipsilateral arm is almost inevitable after axillary clearance. The development of oedema has been linked to a number of factors, which also include surgical technique. Segerstrom et al found an increased incidence of lymphoedema after mastectomy when an oblique incision was used. ${ }^{4}$ Larson et al have also attributed the risk of developing arm oedema to the extent of axillary dissection that was done. They reported an incidence of $37 \%$ for those who underwent full dissection compared to only $8 \%$ for those who just had lower axillary nodal clearance. ${ }^{2}$ However, others like Hoe have reported that full axillary clearance did not lead to higher incidence of arm lymphoedema. In their series of 118 patients of breast cancer who underwent full axillary clearance along with either mastectomy or lumpectomy, only 9 patients (7.6\%) developed lymphoedema. ${ }^{5}$ Other than the extent of axillary surgery, other factors which have been implicated in the development of arm lymphoedema include number of involved axillary nodes, radiation to the axilla and obesity. Some studies have also implicated other conditions like operation on the dominant arm and previous infections of the arm as a risk factor for lymphoedema development. ${ }^{6}$ However, other workers have not found these to be significant issues. Pillai et al found axillary radiation and the number of positive axillary lymph nodes to be the only two factors responsible for development of lymphoedema, in a prospective study from India. ${ }^{7}$ Our study also analysed a few common factors which have been implicated in the causation of lymphoedema after axillary dissection. However, our premise based on our own experience is that significant lymphoedema of the arm is quite uncommon after axillary surgery although minor increase in the circumference of the arm or forearm is not uncommon. We believe this is due to the preservation of the axillary fascial covering over the anterior surface of the axillary vein through which the arm lymphatic channels pass. In all our patients who were operated upon by two surgeons, the same technique of preservation of the fascia over the axillary vein was meticulously adhered to. As a result, the incidence of lymphoedema in our series was not very high (25.5\%) and none of them developed significant oedema within the follow up period. This is in tune with the incidence reported by other Indian cancer institutes. ${ }^{7,8}$ In world literature, the incidence of lymphoedema reported varies widely. This is because there is no standard definition of lymphoedema and no universally accepted method of measurement of arm lymphoedema. Most widely accepted method of measurement is by evaluating the change in limb volume, which is best done by water displacement method after limb immersion. ${ }^{9}$ However, even this is not universally reproducible and is cumbersome and not widely available. Taylor and his co-workers validated the method of assessing limb volume by measurement of arm circumference from fixed bony landmarks compared to volume displacement method and found it to be accurate and reliable. ${ }^{10}$ Hence we used measurement of limb circumference in our study as well.

lour study has some drawbacks. Lymphoedema is an ongoing process and some patients who have mild oedema at 1 year may progress to severe oedema on long term follow up. So a longer follow up period would demonstrate the success of our technique in preventing lymphoedema more convincingly. Secondly, the patients in our study were compared to their own baseline values of arm volume. A cohort study with another group of age and disease matched patients undergoing Level 11 dissection only as a comparator would make the study more robust. However, as an initial study documenting the low incidence of lymphoedema using a particular operative technique, our study is important.

\section{REFERENCES}

1. Pain SJ, Purushotham AD. Lymphoedema following surgery for breast cancer. Br J Surg 2000; 87:1128-41.

2. Larson D, Weinstein M, Goldberg I, Silver B, Recht A, Cady B, Silen W, Harris JR. edema of the arm as a function of the extent of axillary surgery in patients with stage l-ll carcinoma of the breast treated with primary radiotherapy. Int'l $J$ of Radiation Oncol Biol Phys 1986; 12: 1575-82.

3. Markowski J, Wilcox JP, Helm PA. Lymphoedema incidence after specific post-mastectomy therapy. Arch Phys Med Rehabilitat 1981; 62: 449-52.

4. Segerstrom K, Bjerle P, Graffman S, Nystrom A. Factors that influence the incidence of brachial oedema after treatment of breast cancer. Scand J Plast Reconstructr Surg Hand Surg 1992; 26: 223-7.

5. Hoe AL, Iven D, Royle GT, Taylor I. Incidence of arm swelling following axillary clearance for breast cancer. Br J Surg 1992; 79: 261-62.

6. MakSS, Yeo W, Lee YM et al. Predictors oflymphedema in patients with breast cancer undergoing axillary lymph node dissection in Hong Kong. Nurs Res 2008; 57: 416-25.

7. Pillai PR, Sharma S, Ahmed SZ, Vijaykumar DK. Study of incidence of lymphedema in Indian patients undergoing axillary dissection for breast cancer. Ind J Surg Oncol 2010; 1: 263-9.

8. Deo SVS, Ray S, Rath GK et al. Prevalence and risk factors for development of lymphoedema following breast cancer treatment. Ind J Cancer 2004; 41: 8-12.

9. Megens AM, Harris SR, Kim-Sing C, McKenzie DC. Measurement of upper extremity volume in women after axillary dissection for breast cancer. Arch Phys Med Rehabil 2001; 82:1639-44.

10. Taylor R, Jayasinghe UW, Koelmeyer L, Ung O, Boyages J. Reliability and validity of arm volume measurement for assessment of lymphoedema. Phys Ther 2006; 86: 205-14. 